\title{
Typologie des précipitations quotidiennes, mensuelles et saisonmières sur la France
}

Classification of daily, monthly and seasonal rainfall over France

\author{
G. Der Megreditchian, A. De Billy, R. Foka, J. Y. Grosse et M. C. Rullière \\ Météorologie Nationale, Paris
}

\section{Introduction}

L'étude climatologique du fichier de précipitations sur la France déborde le cadre de la statistique descriptive se résumant à calculer divers paramètres exprimant les différents aspects du comportement moyen du champ de pluie. En effet, dans une approche globale de ce type, on ne tient pas compte de la non homogénéité du fichier. Les précipitations ont des origines diverses et correspondent à des situations météorologiques différentes. Dans une telle étude statistique globale, on mélange des cas dissemblables, car on dispose non pas d'une population statistique unique dont on a extrait un échantillon, mais d'un fichier de données obtenu à partir de populations différentes. Une étude plus fine consistera à décrire ces populations à partir d'un traitement adéquat de la réponse du phénomène pluie à l'évolution de la situation météorologique. L'ensemble des cartes disponibles des précipitations sera scindé en classes définissant les types de répartition spatiale de la pluie. L'outil statistique approprié est évidemment la Classification Automatique. Elle sera effectuée à trois niveaux correspondant ̀̀ une certaine concrétisation temporelle du fichier de base suivant un découpage quotidien, mensuel et saisonnier.

\section{Méthodologie de l'étude}

\section{Le fichier disponible}

Le matériel de base est le fichier pluviométrique de la France élaboré au Bureau de l'Eau de la Météorologie Nationale, c'est-à-dire l'ensemble des observations $x_{i}(t)$, valeurs de la pluie journalière relevées à la station numéro $i$ pour le jour numéro $t$; les plages de variation des indices sont les suivantes : $i \in(1, N), t \in(1, T)$ où $N$ désigne le nombre total de stations et $T$ le nombre total de jours. Le fichier original est donc un être spatiotemporel puisqu'il donne l'image de l'évolution temporelle de la répartition spatiale de la pluviométrie sur la France. Cette réprésentation spatio-temporelle est évidemment trop riche (elle comporte 18 années d'obser. vations sur environ 3000 stations), pour être assimilée directement. On peut en tirer certaines images plus concrètes, en définissant deux caractéristiques globales de la pluviométrie.

a) Pour le jour $t$, la carte de la pluviométrie journalière de la France définie par le vecteur colonne

$$
X(t)=\left\{x_{1}(t) \ldots, x_{i}(t) \ldots, x_{N}(t)\right\}
$$

des valeurs observées à ce jour pour toutes les stations.

On aura ainsi, pour une date donnée, une image de la distribution spatiale de la pluviométrie.

b) Pour une station $i$ déterminée, l'hitorique de toutes les valeurs observées pendant la période $T$ défini par le vecteur colonne :

$$
x_{i}=\left\{x_{i}(1) \ldots, x_{i}(t) \ldots, x_{i}(T)\right\}
$$

On aura ainsi, pour une station donnée, une image temporelle de la pluviométrie pendant la période considérée de $T$ jours.

\section{Classification des situations météorologiques}

Suivant que l'on adopte comme élément de base du fichier l'une ou l'autre de ces caractéristiques, deux approches peuvent être considérées.

\section{a) Problème de typisation}

On considère le fichier $\mathscr{X}$ comme un ensemble de $T$ cartes journalières

$$
\mathscr{C}=\{X(1) \ldots, X(t) \ldots, X(T)\}
$$


On désire effectuer une typisation de ces cartes, c'est-à-dire partitionner le fichier $\mathfrak{X}$ en classes comportant des éléments semblables.

Autrement dit, nous rangerons dans une même classe, les cartes de pluviométrie journalière ayant la même allure. A chaque classe, on pourra associer une image type de répartition de la pluviométrie constituant un élément représentatif de la classe.

\section{b) Problème de régionalisation}

On considère le fichier comme un ensemble de $N$ historiques des stations considérées.

$$
\mathfrak{X}=\left\{X_{1}^{\prime} \ldots, X_{i}^{\prime} \ldots, X_{N}^{\prime}\right\}
$$

On désire effectuer une régionalisation des stations basée sur une classification de leurs historiques, autrement dit procéder à un découpage de la France en régions ou zones regroupant les stations dont l'évolution temporelle de la pluviométrie est semblable.

Bien entendu, dans les deux cas, la notion de "similitude" doit être précisée, c'est-à-dire que l'on adoptera une certaine distance entre les éléments considérés. Etant donné qu'il s'agit d'une distance entre situations pluviométriques, le choix d'une métrique appropriée est difficile et se place en dehors d'une théorie mathématique; il devrait être guidé par des considérations météorologiques. Les deux problèmes envisagés constituent ainsi deux aspects particuliers du problème général de classification automatique consistant à partitionner un ensemble d'éléments en classes disjointes.

\section{Principe de la classification}

La partition d'un ensemble en sous-ensembles disjoints peut évidemment s'effectuer d'une multitude de façons, mais le principe directeur général s'énonce de la façon suivante : Les éléments appartenant à une même classe doivent être plus "proches" entre eux que les éléments appartenant à des classes différentes.

Le choix de l'algorithme retenu a été conditionné par les particularités du problème concret que nous abordons, résidant entre autres dans l'énormité des fichiers et la nécessité de présenter une image type des classes ainsi formécs. Nous avons rćalisć un algorithme dit méthode des étalons, proposé en 1969 par l'un des auteurs, [1] et s'apparentant à la méthode agglomérative de classification, proposée par MacQueen [2]. Deux options ont été réalisées :

- le nombre de classes est fixé à l'avance ;

-- le nombre de classes n'est pas fixé à l'avance : on doit choisir à la fois, les classes et le nombre de classes.

\section{Caractéristiques du fichier}

La période d'observation a porté sur 18 années (du $1 / 01 / 1961$ au $31 / 12 / 1978$ ) soit $T=6574$ jours, pour un nombre de stations d'environ 3000 ; il est par conséquent impossible de traiter le problème à partir du fichier original.

Une première opération a donc consisté à condenseì l'information disponible de manière à réduire la masse globale des données.

Nous sommes passés d'un système de $N$ stations à un système réduit de $n$ stations généralisées. La station généralisée est simplement une caractéristique de tendance centrale, en l'occurrence la valeur moyenne de la distribution spatiale des précipitations sur une unité de surface qui, dans cette première approche a été prise comme le département. Ce découpage administratif, bien que fort éloigné de considérations pluviométriques présente néanmoins un certain intérêt, en particulier du fait qu'il définit généralement un ordre de grandeur comparable des unités de surface. Dans une étude ultérieure, cette unité de surface sera précisée de manière à obtenir une échelle de classification plus fine et plus réaliste. Par ailleurs, la période considérée de 18 ans est certainement encore insuffisante pour obtenir des résultats définitifs et nous comptons la porter à une période de 30 ans.

\section{L'algorithme de classification}

C'est un algorithme itératif comportant deux variantes : l'algorithme mono-étalon (à chaque classe, est associé un seul étalon) et l'algorithme multi-étalons (à chaque classe, sont associés plusieurs étalons).

\section{Description de l'algorithme}

\section{a) Nombre de classes fixé à l'avance}

Dans ce cas, l'algorithme comporte une première phase dite d'initialisation où l'on effectue un choix "raisonnable" des étalons.

Un étalon est ici tout simplement un point de l'espace des éléments devant être classés dont la dimension sera, suivant l'approche considérée, soit $n$ pour la typisation, soit $T$ pour la régionalisation. Le principe de la méthode consiste, après chaque nouveau classement, à assimiler chacune des classes élaborées à l'étalon de cette classe. Après introduction d'une distance, on pourra affecter un élément non encore classé, à une classe donnée en recherchant l'étalon le plus proche de l'élément considéré. Après chaque nouvelle affectation, on recalcule la matrice des étalons et on classe l'élément suivant.

b) Nombre de classe non fixé :

Dans ce cas, on définit deux paramètres $C_{1}$ et $C_{2}$, où $C_{1}$ désigne la distance maximum d'un élément à l'étalon de la classe à laquelle il appartient et $C_{2}$ la distance minimum entre les étalons de deux classes différentes. Le principe de classification implique l'obtention de classes suffisamment homogènes, donc peu étalées, mais par contre nettement séparées. Le premier élément étant considéré comme le centre ou l'étalon de la première classe, on effectue l'affectation de classe de la même manière que dans le cas où le nombre de classes est fixé à l'avance, mais en tenant compte à chaque pas des contraintes imposées par les paramètres $C_{1}$ et $C_{2}$.

$\mathrm{Si}$ la distance entre un élément et l'étalon qui lui est le plus proche est supérieur à $C_{1}$, une nouvelle classe doit être créée et cet élément en sera le nouvel étalon.

D'autre part, après chaque nouvelle affectation de classe on recalcule la matrice des étalons. Si deux classes sont trop voisines, (la distance qui les sépare est inférieure 
à $C_{z}$ ) on les regroupe en une seule classe. Le nombre de classes peut ainsi varier dans les deux sens.

\section{c) Choix du nombre d'étalons par classe}

Concrétisons maintenant, la notion d'étalon. Si l'étalon de chaque classe est défini par un seul point de l'espace des éléments à classer, la méthode sera dite mono-étalon; les classes correspondantes sont dans ce cas des hyperboules.

Une généralisation immédiate consiste à choisir plusieurs étalons par classe afin d'éliminer la contrainte relative à la forme trop particulière de la classe. Il semble plus naturel d'assimiler les classes à des hyperellipsoïdes. On ne retiendra pas ici le principe de hiérarchisation des étalons potentiels suivant la proximité au premier étalon tel qu'il est adopté, par exemple, pour la méthode des Nuées Dynamiques [3]. Au fil des itérations, cela a pour effet, si le nombre d'éléments à classer est suffisamment élevé, de rapprocher de plus en plus les hyperellipsoïdes de départ d'une hyperboule.

Dans la méthode multiétalons que nous proposons, concrétisée par l'algorithme "ETALON F", nous appellerons étalons de chaque classe, les foyers de l'hyperellipsoïde associée à cette classe. Dans un souci de simplicité, les ellipsoïdes seront des ellipsoïdes de révolution ne comportant que deux foyers. La distance d'un élément à l'étalon de la classe, s'identifie alors à la somme des distances de cet élément aux foyers de l'hyperellipsoïde.

$$
D(X, A)=D(X, F 1)+D(X, F 2)
$$

Les foyers étant obtenus par diagonalisation de la matrice correspondante, la procédure "ETALON F" est évidemment beaucoup plus lourde que les procédures "ETALON 1" - "ETALON 2" - "ETALON 3" réalisant les algorithmes proposés et que nous décrirons plus loin, mais par contre, les classes obtenues seront certainement plus réalistes.

Pour atténuer, dans la mesure du possible, la subjectivité introduite par l'initialisation "raisonnable" adoptée, nous effectuons d'abord une itération complète en classant successivement tous les éléments du fichier. Après cela, on recommence la classification des éléments en utilisant la matrice des étalons, obtenue à la fin de la première itération.

Le critère d'arrêt des itérations est celui de la stabilité des résultats obtenus pour deux itérations successives. Si le pourcentage d'éléments classés de façon différente au cours de deux itérations successives devient inférieur à un certain seuil choisi à l'avance, on considère que la stabilité est atteinte.

Quatre programmes ont été élaborés pour mettre en œuvre les algorithmes que nous avons décrits.

"ETALON 1", détermine les étalons initiaux jugés "raisonnables".

Le premier étalon correspond au premier élément du fichier. Le second est l'élément le plus éloigné du premier. Les étalons suivants seront déterminés successivement comme les éléments du fichier qui maximalisent la somme des distances aux étalons définis précédemment.

"ETALON 2" réalise le principe de classification automatique exposé précédemment pour le cas du nombre de classes fixé à l'avance. Il utilise pour cela les étalons obtenus par "ETALON 1".

"ETALON 3" réalise la classification automatique dans le cas du nombre de classes non fixé à l'avance. Le choix des paramètres $C_{1}$ et $C_{2}$ ne présente pas de difficulté et s'effectue simplement par une méthode d'essais basée sur la variation de ces paramètres avec un certain pas.

"ETALON F" réalise la classification basée sur l'affectation d'un élément à la classe correspondant au minimum de la somme de ses distances aux foyers des hyperellipsoïdes définissant les classes.

La méthode de classification automatique que nous présentons nous a semblé particulièrement bien adaptée à l'objet de son application, mais il faut noter que ce domaine reste un centre d'intérêt pour de nombreux chercheurs dans le monde et qu'en France, en particulier, différentes méthodes ont été proposées, la plus populaire étant certainement celle des Nuées Dynamiques élaborées par Diday E. [3].

Ces programmes ont été mis au point sur un Control Data 175. "ETALON 1" - "ETALON 2" sont toujours exécutés successivement pour un même passage en machine.

\section{d) Qualité de la classification}

D'un point de vue qualitatif, le problème de la classification a été đéfini comme le problème de la partition d'un ensemble fini d'éléments en classes disjointes, de manière à ce que les éléments appartenant à une même classe soient le plus ressemblants possible et les éléments appartenant à des classes différentes soient le plus dissemblants possibles. Ces notions doivent être précisées. Tout d'abord, il faut introduire une métrique dans l'espace des observations pluviométriques. Ce choix, d'une grande importance, est difficile, car il existe fort peu de recommandations à ce sujet.

Nous avons adopté une voie pragmatique consistant à essayer successivement plusieurs métriques connues en commençant bien entendu par la plus utilisée, la métrique euclidienne, et à dégager une métrique "optimale" pour le problème et le fichier considérés à partir de certains critères de qualité de la classification obtenue.

On trouvera dans [4], le détail de la définition de la métrique, du codage, de la distance entre cartes et entre historiques, les caractéristiques d'homogénéité intra et inter-classes.

\section{Description des résultats}

\section{Classification des cartes quotidiennes}

La Classification Automatique a été appliquée au champ des précipitations journalières pour un nombre de classe $k=10$ et $k=20$. La convergence de l'algorithme est assez rapide ( 9 itérations).

Quelques étalons des classes de pluie quotidienne sont donnés sur la figure (1A). Les classes obtenues présentent un certain intérêt météorologique. La classe 1 (47\% des cas) correspond aux situations d'absence de pluie ou de pluies trés faibles, c'est-à-dire aux types de temps anticycloniques, en particulier. 


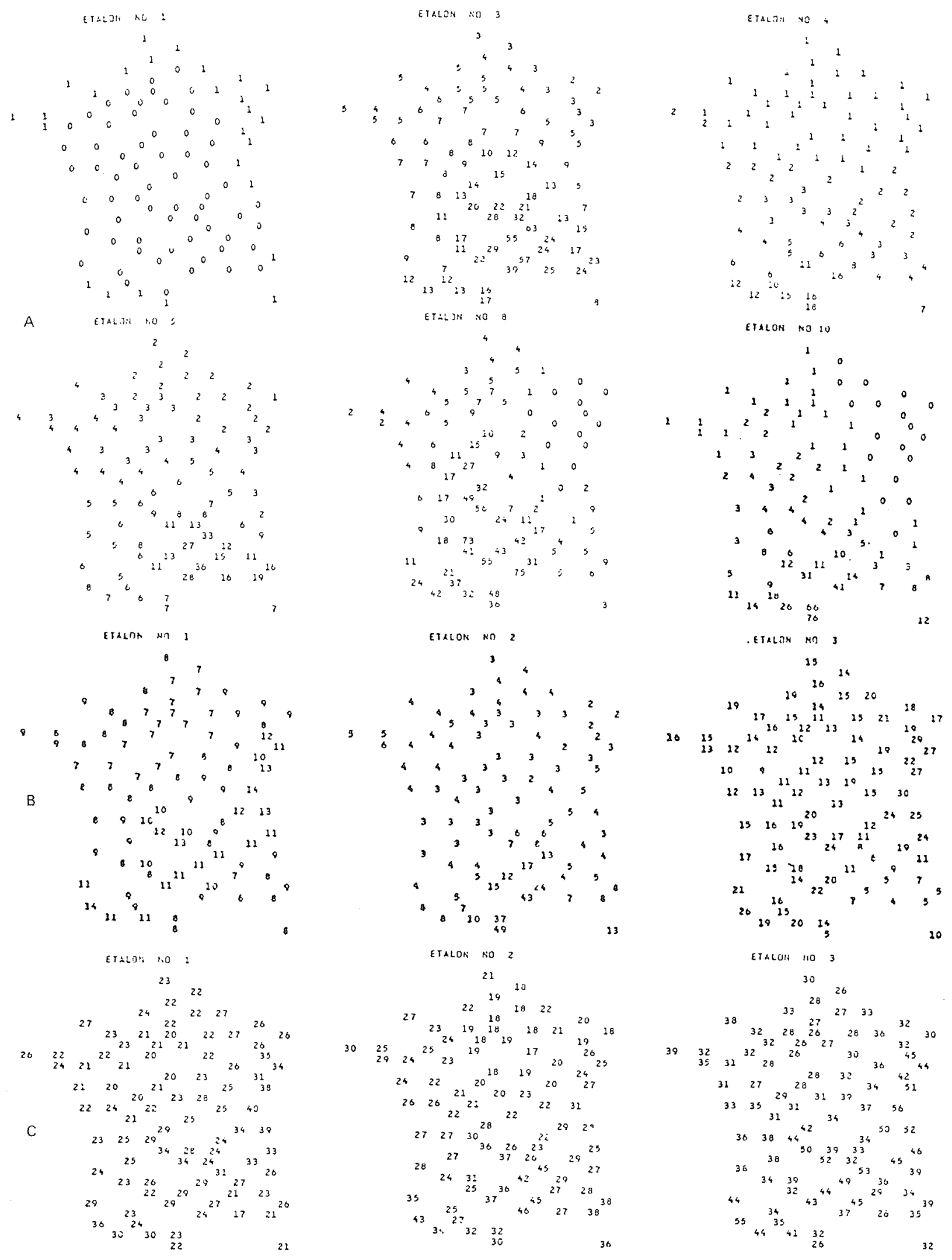

Figure $1 \mathrm{~A}$, B et $\mathrm{C}$ - Etalons des classes de répartition spatiale des précipitations obtenues sur le fichier des pluies quotidiennes ( $1 \mathrm{~mm}$ ), mensuelles $(10 \mathrm{~mm})$, saisonnières $(10 \mathrm{~mm})$. 
Les classes 10 et 4 présentent une configuration pluviométrique similaire, quoique d'intensité différente. Il s'agit de situations plutôt d'automne et d'hiver. Les pluies se situent sur le Sud de la France et surtout sur l'Est des Pyrénées et l'Herault. Dans ce genre de situation pluviométrique, la France est soumise à un flux de secteur Sud, provoqué, soit par une zone dépressionnaire allant de l'Islande au Sud de l'Europe et se déplaçant vers l'Est et le Sud-Est, soit par des perturbations sur l'Espagne. Les pluies peuvent être fortes et localement orageuses.

Les classes 3 et 8 voient les fortes pluies se situer dans la moitié Sud de la France et surtout sur le Massif Central Sud. On est certainement dans ce genre de situations en présence d'un anticyclone situé vers le relief alpin ou l'Europe Orientale. Ce dernier est un facteur d'accentuation des pluies sur le Massif Central (effet de blocage), face au courant perturbé d'Ouest. Dans la classe 8, l'effet de blocage est plus marqué à l'Ouest.

Pour la classe 5, la zone de pluie qui va des Cévennes aux Alpes Maritimes ne déborde pas sur les Alpes du Nord. Le flux de Sud-Est est avec averses et orages sur la Méditerranée.

Une classification plus détaillée serait certainement souhaitable avec un tel fichier pour un nombre plus important de classes afin d'affiner cette étude des types de temps. Rappelons à cet égard, que sur un fichier de 4017 observations, un tel travail a été réalisé [5] en faisant varier le nombre de classes de 10 à 100 .

Un aspect important de la climatologie dynamique du champ de précipitation est contenu dans le tableau I décrivant l'évolution temporelle des types à partir duquel on peut calculer les probabilités de passage de type à type, étudier la persistance et la fréquence des types. On présente sur la figure $2 \mathrm{~A}$ des exemples de probabilités de passage type à type.

L'analyse fréquentielle des types concrétisée par le tableau II nous donne les fréquences absolues d'apparition de chaque type par année et par mois. Cela permet en particulier la caractérisation d'une année ou d'un mois par la fréquence de chacun des types.

Pour une période donnée, l'entropie des fréquences relatives correspondantes nous apporte une appréciation quantitative de la non uniformité de ces fréquences. Par exemple, la sècheresse étant définie par la prédominance des types "secs", cela correspond à une entropie minimum (voir les mois de juin 1976, septembre 1977 , octobre et novembre 1978).

\section{Classification des cartes de pluies moyennes mensuelles}

Le schéma général de l'étude est analogue à celui des cartes quotidiennes. La classification automatique a été réalisée avec les 2 programmes "ETALON 2" et "ETALON F" en faisant varier le nombre de classe de 4 à 9. Le nombre d'éléments à classer est ici égal à 216 . Nous avons retenu un exemple avec $k=6$ classes.

Les étalons des classes des pluies mensuelles sont donnés sur la figure $1 \mathrm{~B}$. De même, on trouvera dans [6], l'analogue du tableau I décrivant l'évolution chronologique de l'affectation des classes. Les probabilités de passage de type à type sont données sur la figure $2 \mathrm{~B}$.

Sans détailler la description des classes correspondantes, nous présenterons l'utilisation de la classification obtenue dans un esprit prévisionnel.

Tableau I. -- Chronologie d'affectation des types de précipitation pour le fichier des pluies quotidiennes.

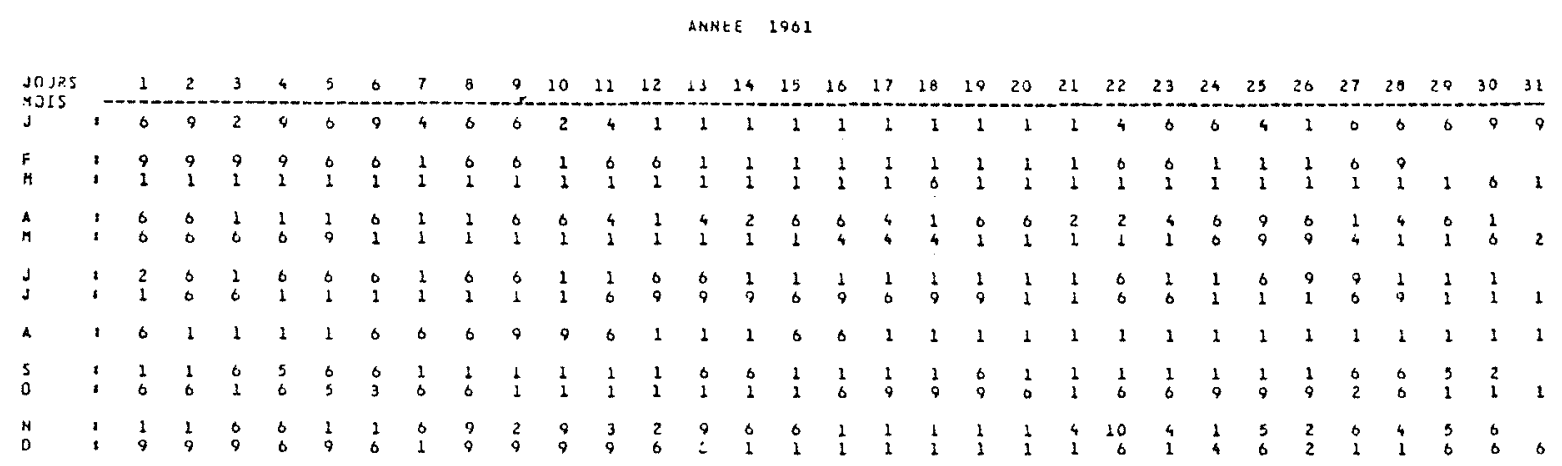

Tableau 11. - Fréquences absolues des types de pluie quotidienne calculées pour chaque année.

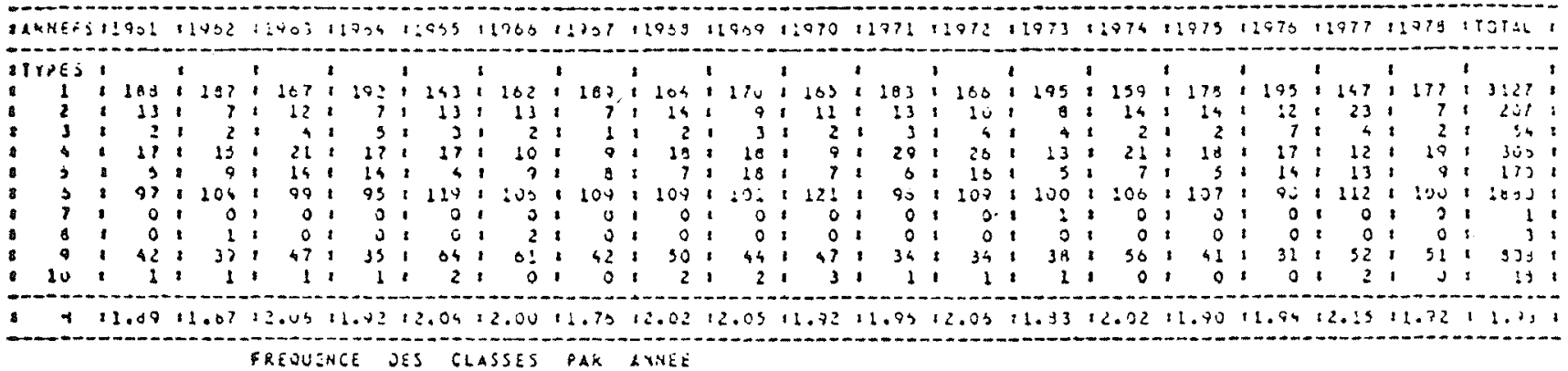



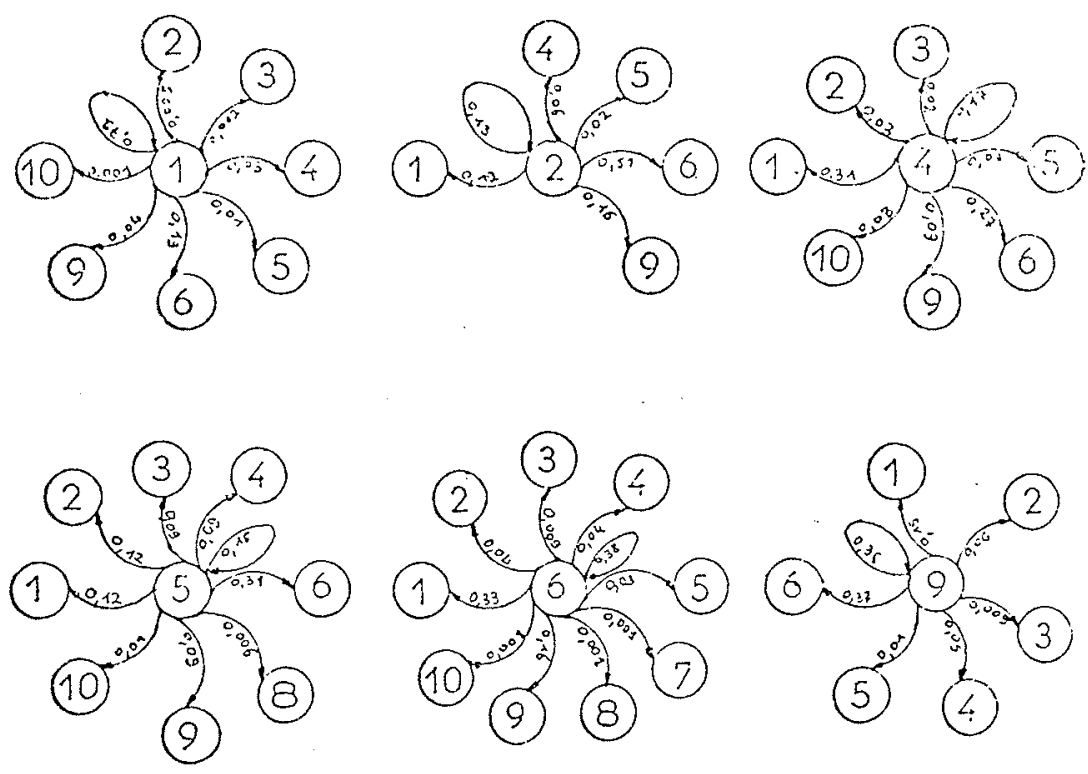

Figure $2 \mathrm{~A}$ - Probabilités de passage de type à type pour les pluies quotidiennes.
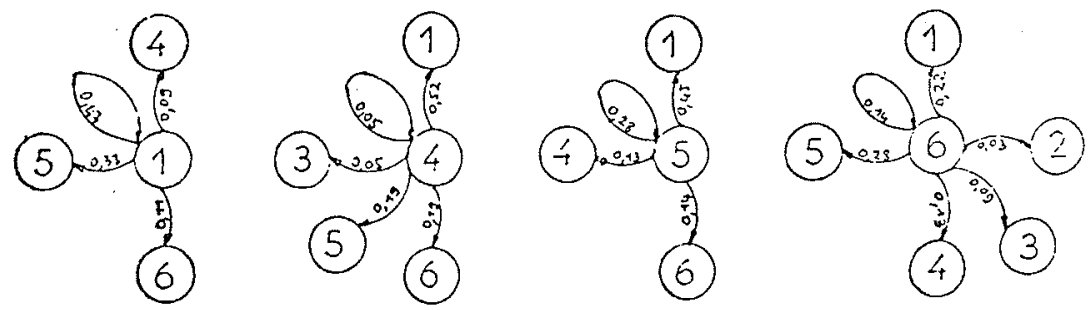

Figure 2B - Probabilités de passage de type à type pour les pluies mensuelles.
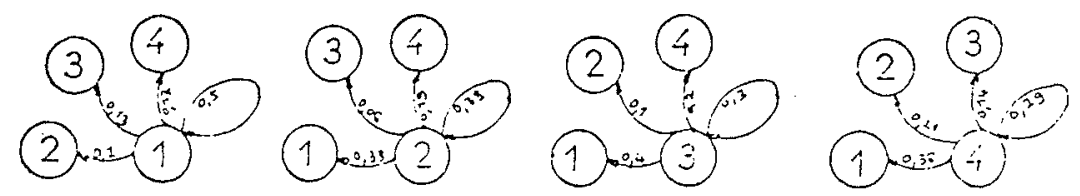

Figure $2 \mathrm{C}$ - Probabilités de passage de type à type pour les pluies saisonnières.

\section{Analyse factorielle des correspondances synchrones entre types de champ prédictand et type de champ prédicteur}

IOn a considéré la correspondance entre les types de champ prédictand et les types de champ prédicteur (géopotentiel 500 mbar et température), obtenus par le programme "ETALON F", pour $k=5$ classes.

a) Analyse factorielle des correspondances synchrones entre types de champ de précipitation et de géopotentiel

Le champ prédicteur est ici le champ moyen mensuel de géopotentiel 500 mbar défini sur la demi hémisphère Atlantique, de la Californie à l'Oural, pour la période janvier 1963-avril 1978, sur une sous-grille du fichier PANAL (1 point sur 3, soit 104 points). Dans cette étude (Fig. 3), seuls les deux premiers axes sont significatifs : le premier axe explique $q_{1}=47,16 \%$ et le deuxième $q_{2}=35,27 \%$ de l'inertie totale.

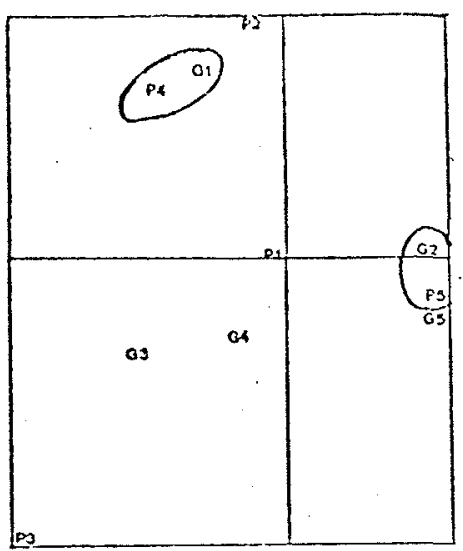

Figure 3 - Analyse factorielle des correspondances entre types de précipitation (codés $P I$ ) et types de champ de géopotentiel (codés $G J$ ). 
- Le premier axe factoriel associé au "type sec" classe les différents types suivant un ordre presque naturel, défini par l'opposition des types correspondant aux mois d'été, $P 5$ et $G 2$ et ceux correspondant aux mois d'hiver $P 3$ et $G 3$.

- le deuxième axe factoriel associé au "type arrosé" correspondant aux types $P 3, P 4$ et $G 1$ (mois de novembre).

On note une certaine continuité des types de précipitations sur le graphique : on passe du type "bien arrosé" au type "sec" en passant par un type "moyennement arrosé" correspondant aux mois de Mai, Septembre et Octobre : ce sont les mois de transition.

b) Analyse factorielle des correspondances synchrones entre types de champ de précipitation et types de champ de température

Le champ prédicteur est ici le champ de température mesuré en 89 points du demi hémisphère Atlantique Nord pour la période janvier 1961-décembre 1978.

Dans cette étude(Fig. 4). seuls les deux premiers axes sont significatifs; le premier sépare les variables en deux groupes : il associe les types $P 5$ et $T 2$ opposés aux types $P 3$ et $T 1$ Cet axe, c'est l'axe du "temps sec". Le deuxième axe, associe les types $P 2, P 4, T 1$ opposés aux types $P 3$ et $T 3$. C'est l'axe du temps "bien arrosé". Autour du centre de gravité, se situent les types $P 1$ et $T 4$ correspondant au mois d'octobre et mai, (mois de transition).

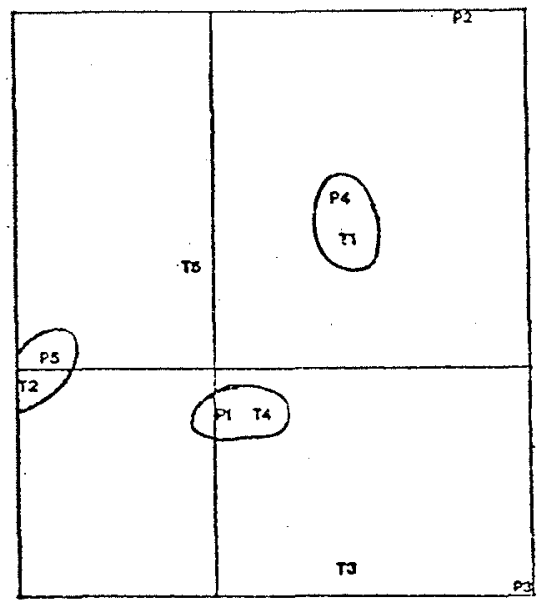

Figure 4 - Analyse factorielle des correspondances entre types de champ de précipitation (codés $P I$ ) et types de champ de température (codés $T K$ ).

c) Analyse factorielle des correspondances synchrones entre les types de champ de précipitation et les types des deux champs prédicteurs (géopotentiel 500 mbar et température)

Ici encore, seuls les deux premiers axes sont significatifs (Fig. 5). Le premier axe représentant plus de $50 \%$ de l'inertie totale sépare les variables en deux classes bien distinctes : la classe formée des types $G 1, P 4$ et $T 1$ d'une part et la classe formée des types $G 2, P 5$ et $T 2$ d'autre part. Le second axe fait ressortir la classe formée

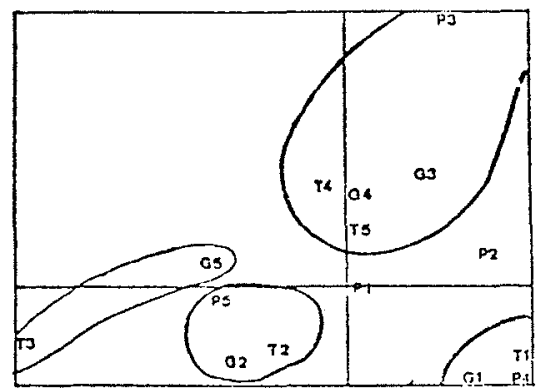

Figure 5 - Analyse factorielle des correspondances entre types de champ de précipitation (codés $P D$ ), types de champ de géopotentiel (codés $G J$ ) et types de champ de température (codés $T K$ ).

des types $G 3, G 4, P 3$ et $T 4$. La représentation graphique, complétée par l'information apportée par les contributions et les cosinus correspondants nous permet d'associer aux types $P 1$, les types correspondants $G J$ et $T K$, que l'on a mis en évidence sur les figures 3,4 et 5 .

Le type P5 est caractéristique du semestre "chaud"; c'est le type le moins "arrosé".

Ce type est associé aux types $G 2$ et $T 2$ caractéristiques des mois d'été : juin, juillet, août. Les types $P 3$ et P4 les plus "arrosés" se rencontrent surtout à l'automne et parfois l'hiver. Ils sont associés aux types $T 1$ et $G 1$ caractéristiques des mois d'avril et novembre ou aux types $T 4$ et $G$ qui sont des mois de transition (essentiellement mai et octobre).

\section{Classification automatique des cartes saisonnières}

Le fichier des cartes saisonnières comporte 71 éléments et nous avons choisi $k=4$ classes pour illustrer cette étude. Les étalons sont donnés sur la figure $1 \mathrm{C}$ et les probabilités de passage de type à type sur la figure $2 \mathrm{C}$.

\section{Conclusion}

Dans cette étude, la méthodologie de la classification automatique est d'abord utilisée pour affiner la climatologie du champ de précipitation, concrétisé au niveau quotidien, mensuel et saisonnier. Cela nous permet de surmonter l'obstacle que représente la grande diversité des cartes de répartition spatiale des précipitations en ramenant l'étude à un nombre modéré de distributions types (cartes étalons); chaque carte est alors affectée à l'un ou l'autre de ces types. On peut ensuite effectuer différentes analyses : analyse markovienne, fréquentielle, analyse factorielle des correspondances entre champs prédicteurs et champ prédictand, analyse discriminante multiclasses sur prédicteurs qualitatifs, etc.

La typologie des champs de précipitation intervient $i c i$, non comme une fin en soi (ce qui n'aurait qu'un intérêt climatologique descriptif), mais comme une étape transitoire, nécessaire pour permettre une étude de type exogène reliant le champ de précipitations à différents champs prédicteurs. 


\section{Bibliographie}

[1] DER-MEGREDITCHIAN G. - Méthodes statistiques de contrôle, d'analyse et de prévision des champs météorologiques. (Thèse de doctorat, Moscou, 1969).

[2] Mac-QUEEN. - Some methods for classification and analysis of multivariate observations. Proc. Fifth, Berkeley Symp. Math. Stat. and Prob., 1967.

[3] DIDAY E. - Une nouvelle méthode en classification automatique et reconnaissance des formes, la méthode des Nuées Dynamiques. R.S.A., 19, 1971.
[4] DER-MEGREDITCHIAN G., RULLIERE M.C. - Classification automatique de la pluiviométrie en France. La météorologie, Série $n^{\circ} 1$, Paris, juin 1975.

[5] DE BULLY A. - Essais de classification automatique sur la France des données pluiviométriques dans l'espace et dans le temps. Thèse de $3^{\mathrm{e}}$ cycle, Paris 1978.

[6] RULLIERE M.C., DER MEGREDITCHIAN G., FOKA R., GROSSE J.Y., DE BILLY A. - Typologie des précipitations quotidiennes mensuelles et saisonnières sur la France. C.R. du Comité Technique 118 SHF sur les Précipitations Atmosphériques, (3-4 juin 1981).

\section{Discussion}

Le Président. - Je remercie Mme RULLIERE pour cet exposé sur un travail particulièrement magistral de l'équipe spécialisée de la Météorologie, et je vais demander si quelqu'un a des questions à poser.

M. MIQUEL. - Je voudrais savoir si les classes que l'on obtient sont stables, ou bien si elles dépendent de l'échantillonnage ? Par exemple, après découpage de l'échantillon, les éléments communs à deux sous-échantillons se retrouvent-ils dans la même classe ? Auquel cas ces classes auraient une certaine réalité

M. DER MEGREDITCHIAN. - C'est un exercice qu'on ne peut pas faire très souvent étant donné les temps de la machine. On l'a fait deux fois.

Mme RULLIERE. - On a travaillé sur un fichier qui comportait d'abord les années 1961 à 1971 puis 1971 à 1978. On constate, en effet, que les classes sont stables et que l'on retrouve les même configurations. Il y a une chose dont je n'ai pas parlé cela concerne la classe $n^{\circ} 7$, qu'on a obtenue ici. Cette classe ainsi créée correspond à un jour très particulier de 1973 qui ne figurait donc pas dans le premier fichier: elle est très particulière parce que ce sont des cartes maritimes, on a $123 \mathrm{~mm}$ de moyenne départementale pour un jour. Donc, cette carte est évidemment sortie toute seule, alors que les autres classes se retrouvent d'un fichier à l'autre.

M. P.A. ROCHE. - Je voudrais poser la même question que M. MIQUEL, mais concernant les possibilités de passage d'un type à un autre. Vous avez parlé d'impossibilité d'un tel phénomène pour les classes que vous avez définies. Si cela existait à l'échelle mesuelle, ce serait très intéressant, cela voudrait dire qu'on pourrait partir du moi de mai et arriver au mois d'août ? Est-ce que, pratiquement, vous accordez un peu de réalité à cela?

Est-ce que vous envisagez à long terme de faire des prévisions sur les possibilités de types de temps que vous aurez et à quel niveau pouvez-vous le faire?

M. DER MEGREDITCHIAN. - C'est précisément ce qui justifie l'idée de cette étude; on partait d'un certain nombre de situations (4000); une étude markovienne avait permis d'évaluer les probabilités de transition avec un décalage de trois pas de temps; elle avait été réalisée sur l'ancien fichier : le résultat correspond au dessin écologique où les fleurs représentent les probabilités de passage d'un type à un autre avec, un pas d'unité. Nous avons repris ici cette icée d'une étude markovienne mais en conservant la description, c'est-à-dire la climatologie du fichier pluviométrique. II ne s'agit pas obligatoirement de prendre un nombre de classes très faible. Il faut se reporter pour cela à la thèse d'Ariane DE BILLY où une grande variété de types a été recherchée, un certain optimum se situant autour de 80 , alors que des classifications correspondant à un nombre de classes égal à 100 et plus ont été réalisées.

Ici, il s'agit, d'une part de dépasser l'hypothèse d'une seule population, mais, d'autre part, de ne pas créer un trop grand nombre de classes afin de ne pas être trop lié aux particularités d'échantillonnage du fichier. Dans une première étape, on a considéré que 10 classes était un nombre suffisant.

M. P.A. ROCHE. - Je voudrais poser une autre question. Vous avez décrit un peu chaque type de temps que vous preniez en compte, mais vous n'avez pas parlé tellement des variables qui séparaient ces types, c'est-à-dire que, pratiquement, ils sont discriminés sur quelques variables plus particulièrement ?

M. DER MEGREDITCHIAN. - Cette étude est très détaillée dans la thèse d'Ariane DE BILLY. Ici nous devons tenir compte des possibilités pratiques. D'ailleurs, Ariane DE BILLY, qui est présente, pourrait répondre elle-même, car elle a fait une étude très poussée dans ce sens.

Mlle DE BILLY. - Mon étude est un peu différente. J'ai commencé l'étude au bureau de M. TRENDEL. Notre idée était d'essayer d'associer les photos satellite et les répartitions des qualités de pluie sur la France. Au bureau de l'Eau, on disposait d'un énorme fichier et nos moyens n'étaient pas du tout à la mesure de faire le même type de traitement sur un fichier de photo satellite. On a donc abandonné cette partie du travail. On pourrait peut-être la reprendre.

Notre idée était d'essayer de trouver des liaisons entre la pluie, la température et le géopotentiel. Personnellement. j'a travaillé sur la pluie essentiellement. Mais, si l'on en reste là, il est vrai que ce genre d'étude n'est pas du tout suffisant; il faut absolument traiter les autres facteurs hydrologiques et essayer de trouver des correspondances entre ces variables.

J'avais fait une étude sur 80 types. D'une façon très nette on retrouve la liaison avec tous les types "cévenols", dont il a été question dans la communication de ce matin. En réalité, il n'y a pas de type cévenol; au niveau des quantités de pluie, on retrouve les situations aussi bien sur les Alpes que sur les Pyrénées que sur le nord-est également, et bien sûr, aussi, par exemple, le micro-climat vendéen. Avec 80 types, on a tout de même de très grandes possibilités.

L'étude que j'ai faite était manuelle, à partir des résultats de classification, et je pense qu'il faudrait faire des corrélations avec les autres variables.

M. DERI. - Personnellement je trouve que la typologie est absolument intéressante et importante.

Pour contribuer à cette méthodologie, j'essayerai de conseiller deux choses : il y a un modèle stochastique développé par $\mathrm{M}$. BERNIER, d'EDF, qui traite de la probabilité de concomitance des évènements, et je pense que cette concomitance des types pourrait contribuer à la typologie.

D'autre part, il sera utile d'essayer d'utiliser la fonction de répartition de Poisson, car je suppose qu'il y aura une concomitance à simuler à l'aide de la fonction en question. Je pense qu'on pourrait essayer utilement cette fonction.

Le Président. - S'il n'y a pas d'autres interventions, nous allons arrêter ici la discussion. Nous remercions Mme RULLIERE et M. DER MEGREDITCHIAN de leur brillante communication. 